\title{
Bergsturz Amden 1974
}

\section{Einleitung}

Das Gebiet bei Weesen am Nordufer des Walensees wird durch Felsbewegungen immer wieder gefährdet. Größere Bergstürze ereigneten sich in neuerer Zeit in den Jahren 1974 und 1979. Vom wissenschaftlichen Standpunkt aus ist vor allem die Rutschung von 1974 von großem Interesse, weil sie sich durch Vorboten angekündigt hatte, welche die Behörden veranlaßten, Messungen zur Überwachung der Bewegungen ausführen zu lassen, die nun einen recht guten Einblick in die Kinematik und Dynamik der vorgekommenen Ereignisse gestatten. Die einschlägigen Meßresultate sind einer Botschaft des Regierungsrates (Botschaft 1973) St. Gallen, sowie einem Kommissionsbericht des großen Rates St. Gallen (Zwischenbericht 1974) zu entnehmen. Herr Professor Jäckli von der ETH Zürich hat die Freundlichkeit gehabt, dem Autor weitere Daten zur Verfügung zu stellen. Berichte in der Tagespresse sowie eigene Begehungen in Begleitung von Herrn Dipl. Geol. W. Kyburz vom Geographischen Institut der Universität Zürich ergänzen die Grundlagen für die vorliegende Arbeit.

\section{Lage, Geologie}

Das Bergsturzgebiet befindet sich westlich von Amden am nördlichsten Ufer des Walensees; es ist in Abb. 1 in einer Planskizze dargestellt.

Ein geologisches Profil ist in Abb. 2 gezeigt. Der darin erwähnte Schrattenkalk gehört zur mittleren Kreide und enthält fossile Austern. Losgelöste Gesteinsmassen (sogenannte Schroppen) bilden instabile Massen.

Der stratigraphisch weiter unten liegende Kieselkalk ist stark zerklüftet (Abb.3). Er wurde in einem Steinbruch (vgl. Abb. 1) abgebaut.

\section{Chronik der Ereignisse}

Es wurden im Gebiet Schafbett-Schwarzberg der Gemeinde Amden seit langer Zeit Felsbewegungen beobachtet. Sie weiteten sich im Herbst 1972 zu einer latenten Bergsturzgefahr aus (Botschaft 1973). Den Winter über wurden Meßbänder für Bewegungsmessungen installiert (siehe Abb.1); eine Alarmorganisation wurde aufgebaut. Über die getroffenen Maßnah-

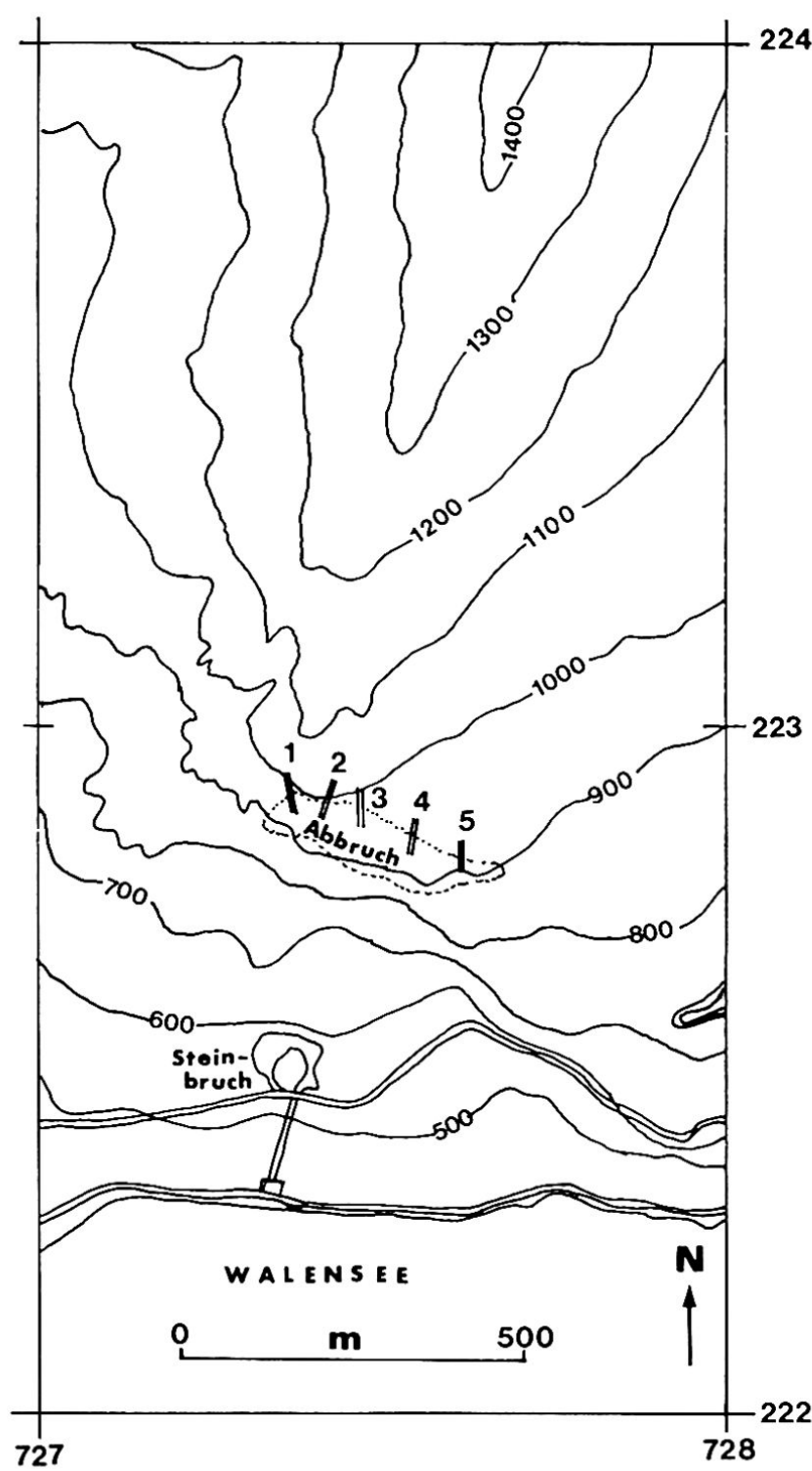

Abb.1 Planskizze des Bergsturzgebietes mit ungefährer Angabe der Bewegungsmeßstellen (Extensometer) No.1-5. Grundlagen: Botschaft des Regierungsrates 1973 sowie persönliche Mitteilungen von Herrn Prof. Jäckli.

A. E. Scheidegger, Prof., Dr., Technische Universität Wien Institut für Theoretische Geodäsie und Geophysik Abteilung: Geophysik

Gußhausstraße 27-29, A-1040 Wien/Austria 


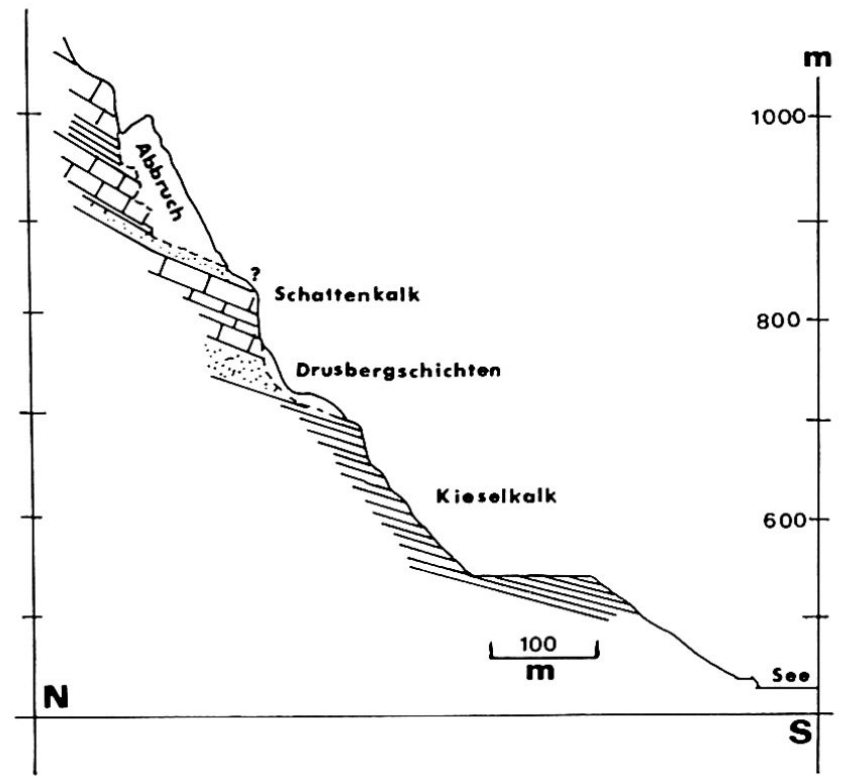

Abb. 2 Geologisches Profil des Bergsturzgebietes. Die in Frage kommenden Schichten gehören durchweg der Kreide an. Grundlagen: Botschaft des Regierungsrates 1973 und eigene Begehungen. men und die eingetretenen Ereignisse wurde laufend in der Tagespresse berichtet.

So brachte die «Zürichsee-Zeitung» am 12. April 1976 eine Meldung über die Einrichtung der Alarmorganisation in Weesen. Am 19. April wurde eine Notiz über die Resultate von Bewegungsmessungen, die Verschiebungen im Zentimeterbereich pro Woche ergeben hatten, und eine Schätzung des Volumens der absturzgefährdeten Felsmassen $\left(500000 \mathrm{~m}^{3}\right)$ gebracht. Während des Restes des Jahres 1973 blieb die Lage mehr oder weniger stationär, aktivierte sich dann aber drastisch nach Mitte Januar 1974. Die kritisch gewordene Lage verschärfte sich schlagartig in der Nacht zum 19. Januar 1974: Die Bewegungen der Meßpunkte stiegen bis auf $4 \mathrm{~cm}$ pro Stunde an, und kleinere Felsmassen (einige hundert $\mathrm{m}^{3}$ ) stürzten auf die Straße von Weesen nach Amden, welche dadurch blockiert wurde (Neue Zürcher Zeitung, 21.Januar 1974). In der Nacht zum 21. Januar 1974 ereignete sich dann ein großer Felssturz, bei dem $55000 \mathrm{~m}^{3}$ Material abstürzten (Zwischenbericht 1974; Neue Zürcher Zeitung, 22. Januar 1974). Eine gleiche Menge Fels blieb «absturzgefährdet». Es wurde vorerst versucht,

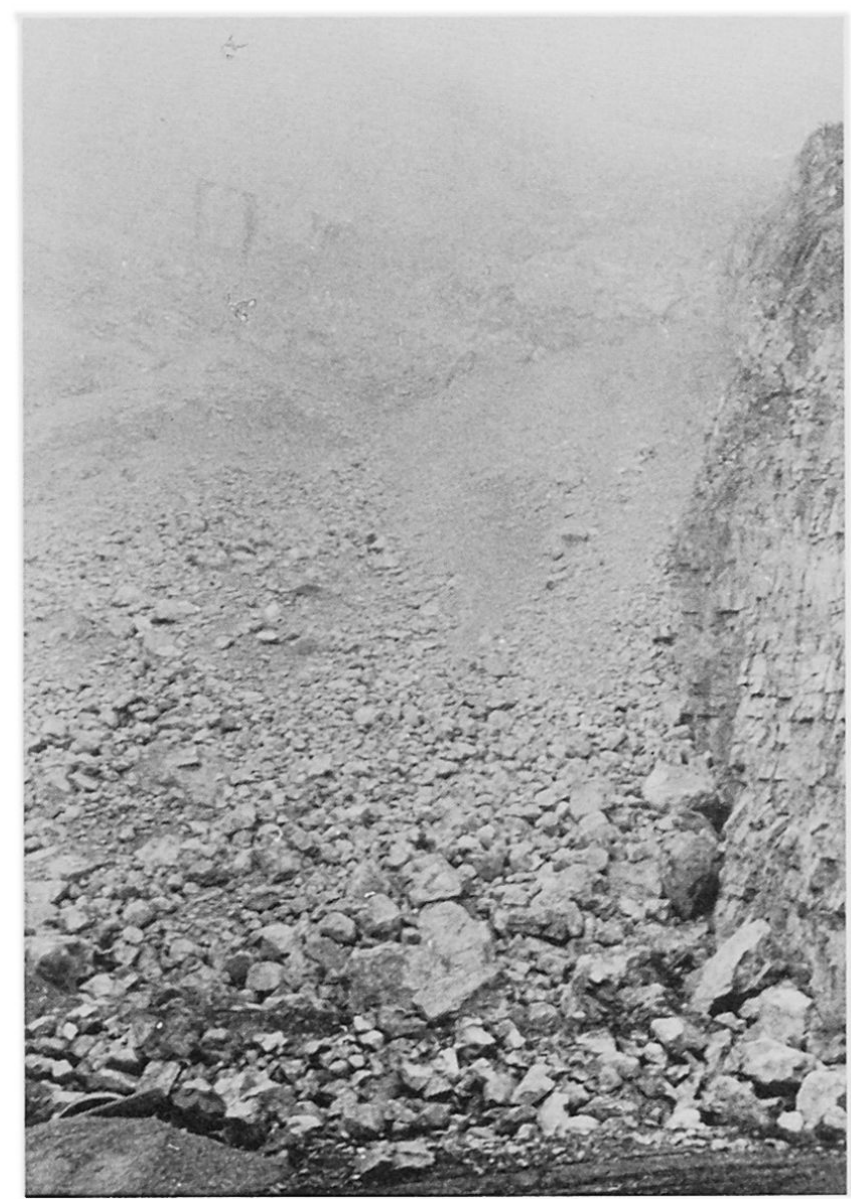

Abb. 4 Bergsturzmasse im Steinbruch, photographiert Anfang Februar 1974. Etwa $55000 \mathrm{~m}^{3}$ Material sind hier liegengeblieben. Scherungen im neotektonischen Spannungsfeld interpretiert, welches aus ihrer Orientierung bestimmt werden kann (vgl. Text!).
Abb. 3 Zerklüfteter Kieselkalk. Derartige Klüfte werden als

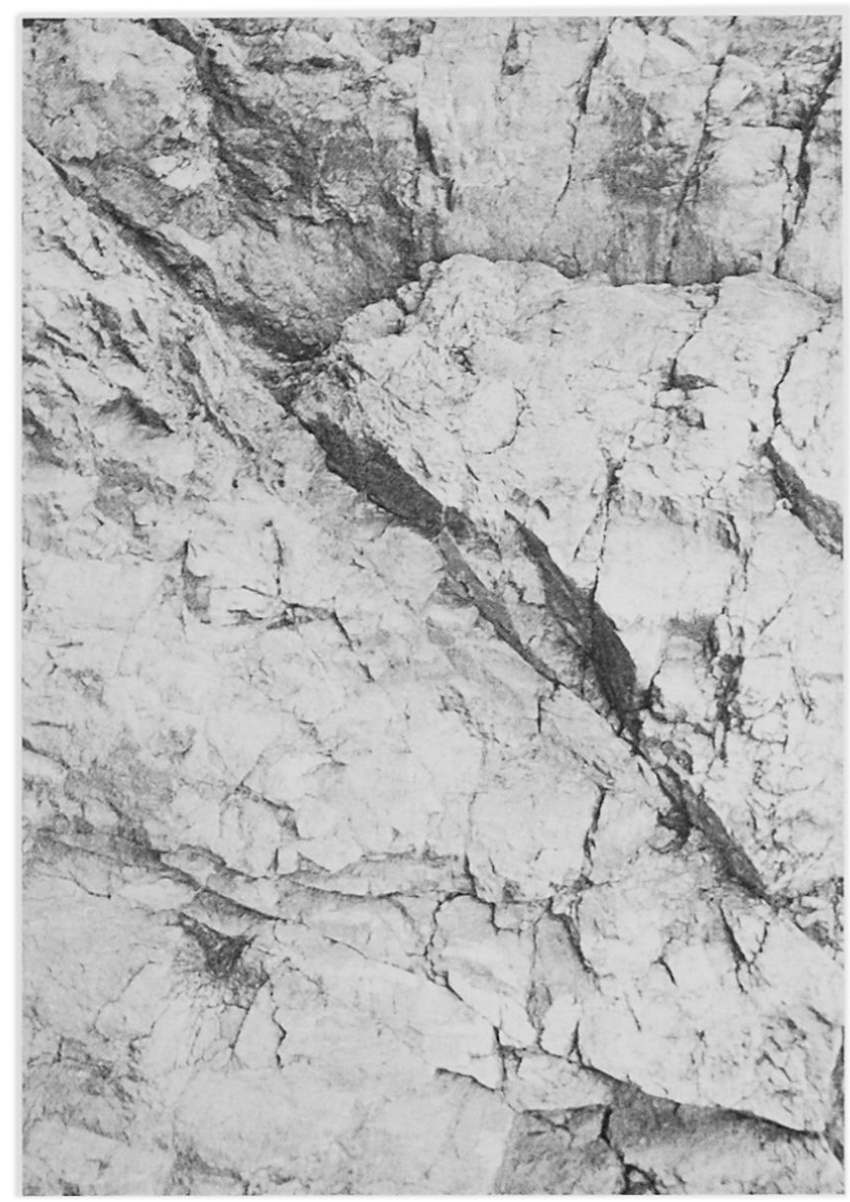




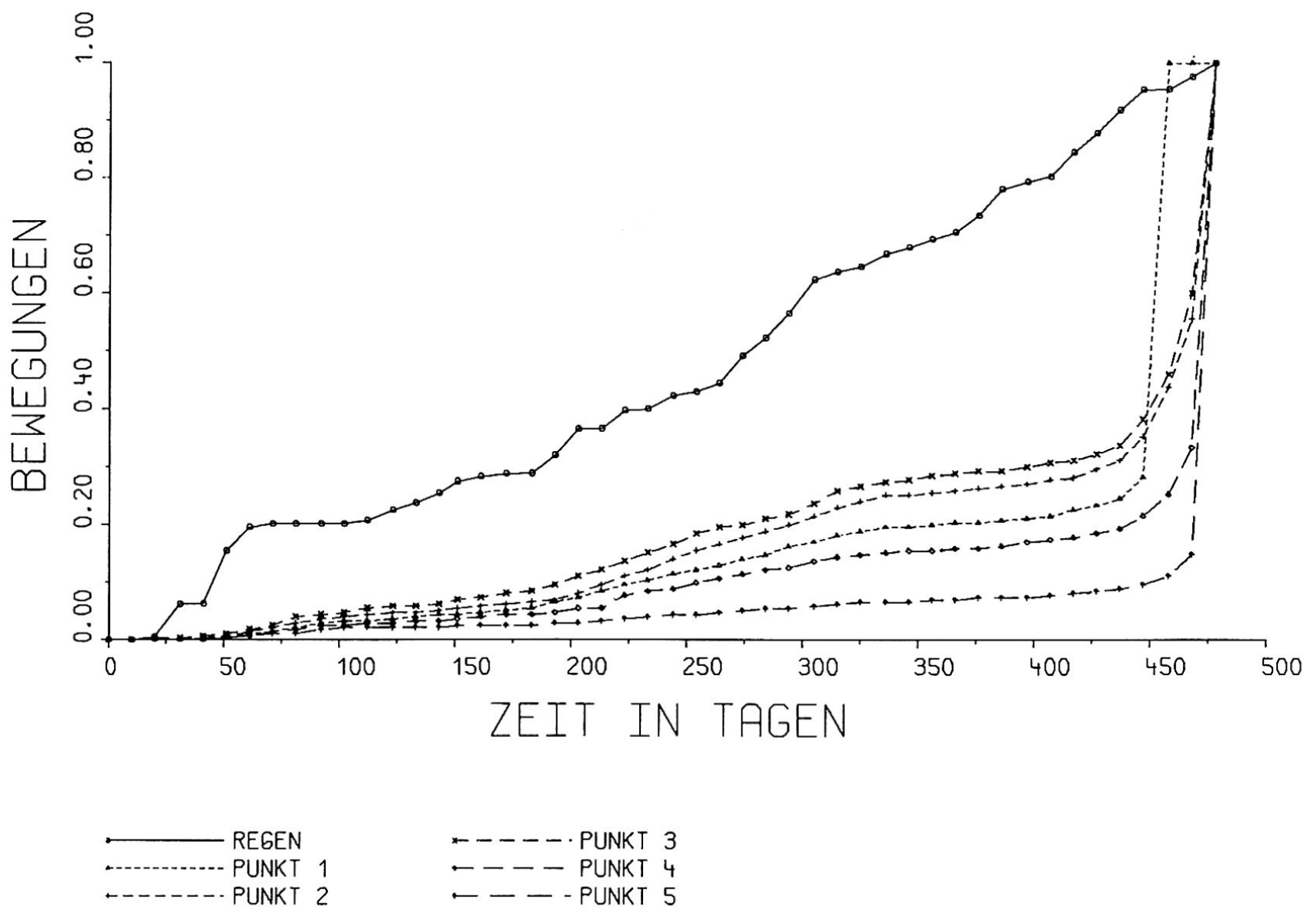

Abb. 5 Bewegungskurven der Meßstellen 1-5 (vgl. Abb.1) “Regen» ist der kumulative Niederschlag seit "Tag 1" (1. Oktober 1972), die Kurven "Punkt 1" bis "Punkt 5" sind die kumulativen Bewegungen an den Meßstellen 1 bis 5 seit Tag 1. Die Endwerte (20. Januar 1974) sind in der Graphik auf «1» normiert; das ist für den Regen gleich $2257 \mathrm{~mm}$ und für die Bewegungen je $270 \mathrm{~cm}$. Man erkennt bis zum Tag 430 (3. Jan. 1974) eine verzögerte Korrelation zwischen Regen und Bewegung; die Rechnung ergibt, daß die Bewegung jeweilen nach 12 Tagen auf den Regen ansprach. Im Januar 1974 wuchs dann die Bewegung exponentiell an, was auf die Ausbildung eines progressiven Bruches hindeutet.

diese durch Wassereinsatz zum Absturz zu bringen (Neue Zürcher Zeitung, 31.Januar 1974), was nur einen geringen Erfolg brachte. Am 8. Februar 1974 wurden schließlich die am meisten absturzgefährdeten Gesteinsmassen, die die Form einer «aufgestellten Birne» hatten, abgesprengt (Tages-Anzeiger, 11. Februar 1974; Die Tat, 11. Februar 1974). Damit war die akute Gefahr gebannt, und man konnte an die Räumung und Öffnung der Straße Weesen-Amden schreiten (Die Tat, 22. Feb. 1974). Es war daher auch dem Schreibenden möglich, das Gebiet am 17. und am 21. Februar $1974 \mathrm{zu}$ besuchen. Das abgestürzte Felsmaterial war zum größten Teil im erwähnten Steinbruch liegengeblieben, wo es nun zu sehen war (Abb.4). Von oben herunterschauend erblickte man die Gleitbahn.

Am Nordufer des Walensees ereigneten sich auch in den folgenden Jahren Rutschungen, so z.B. anno 1979 bei Weesen etwa $1 \mathrm{~km}$ westlich der Amdener Rutschung. Diese hatten aber mit den Amdener Ereignissen von 1974 keine unmittelbare Verbindung (Tages-Anzeiger, 29. Mai 1979).

\section{Kinematik und Dynamik des Bergsturzes}

Dem Bergsturz gingen bergabwärts gerichtete Bewegungen voraus. Die in der Einleitung erwähnten Meßeinrichtungen erlaubten Bewegungskurven von Felsmassen, deren Werte bis Oktober 1973 der Botschaft (1973) entnommen werden können. Bis zum Absturz wurden sie aus einer persönlichen Mitteilung von Prof. Jäckli ergänzt. Da auch Regendaten der Station Weesen von der Meteorologischen Zentralanstalt der Schweiz in Zürich erhoben werden konnten, lassen sich die Bewegungen mit den letzteren vergleichen. Die Meßwerte lagen in Graphiken vor, welche vom Schreibenden zur weiteren Verarbeitung digitisiert wurden. Abb. 5 zeigt eine daraus in Wien erstellte Computergraphik.

Ein einfacher Augenschein dieser Graphik läßt erkennen, daß die Steigung der Regensummenkurve und die Steigungen der Kriechkurven korreliert erfolgen. Eine Korrelationsanalyse ergibt in der Tat eine Andeutung dafür, daß bis zum 3. Januar 1974 (Tag 430) der Regen die Kriechgeschwindigkeit 
beeinflußte, in der Weise, daß die Bewegung jeweilen nach 12 Tagen auf den Regen «ansprach». Am Schluß aber stiegen die Kriechkurven exponentiell an, was auf die Ausbildung eines progressiven Bruches hindeutet.

Schließlich können noch einige Bemerkungen über die Dynamik der Rutschung gemacht werden.

Eine Inspektion der Karte in Abb. 1 zeigt, daß die Masse über eine Horizontaldistanz von $\mathrm{x}=400 \mathrm{~m}$ und durch eine Höhe von $\mathrm{h}=300 \mathrm{~m}$ glitt. Damit ergibt sich der charakteristische Reibungskoeffizient $f$, der den Verlauf eines Bergsturzes bestimmt (sCHEIDEGGER, 1973) zu $\mathrm{f}=\mathrm{h} / \mathrm{x}=0,75$. Dieser $\mathrm{f}$-Wert paßt gut auf die Korrelationskurve des Schreibenden (sCHEIDEGGER, 1973) für eine Masse von $m=55000 \mathrm{~m}^{3}$. Der Amdener Bergsturz entspricht daher genau den Erwartungswerten.

\section{Tektonische Bedingungen}

Um einen möglichen Zusammenhang der Rutschungserscheinungen mit den herrschenden tektonischen Bedingungen festzustellen, wurden Kluftstellungen an 14 Aufschlüssen, die in Abb. 6 mit 1-6 und A-H bezeichnet wurden, gemacht. Wie bekannt (sCHEIDEGGER, 1984) sind die meisten solcher Klüfte als Scherphänomene in einem rezenten Spannungsfeld zu interpretieren. Es lassen sich dann bevorzugte Kluftstellungen statistisch ermitteln; die Winkelhalbierenden der bevorzugten Vertikalstellungen sind die Hauptspannungsrichtungen des sie erzeugenden Spannungsfeldes, wo die Druckrichtung im kleineren Quadranten liegt. Es ist bekannt, daß die größte Druckspannung in der Schweiz in Richtung N $120^{\circ} \mathrm{E}$ liegt; lokal können sich Variationen ergeben (SCHEIDEGGER 1977).

Über die Meßwerte an den einzelnen oben erwähnten Aufschlüssen hat der Schreibende schon einmal berichtet (sCHEIDEGGER, 1977). Um eine etwaige Signifikanz dieser Messungen in bezug auf den Bergsturz aufzuzeigen, wurden die Werte nun noch in anderer Weise, nämlich in Gruppen, ausgewertet. Mit Bezug auf die Lokationen in Abb. 6 wurden 123456, $\mathrm{AB}, \mathrm{CDE}$ und $\mathrm{FGH}$ je als "Gruppe» betrachtet. Die Resultate sind in Abb. 6 aufgetragen.

Zur Interpretation der Resultate sollte man beachten, daß die Stellen 1-3 in der Kalknagelfluh der Speerkette, 4-6 im stark verkarsteten Kalk (untere Kreide) der Mattstock-Kette - beide also im festen Gebirge liegen. Demgegenüber liegen die Stellen A und B genau über dem Abriß des Bergsturzes in Kalk (A) und Schiefer (B), die Stellen CDE auf dem Niveau des Abrisses und FGH an der Straße in der Bahn des Bergsturzes (Kalk).

Eine Inspektion der Abb. 6 zeigt deutlich, daß im untersuchten Gebiet Regionen mit unterschiedlichen,

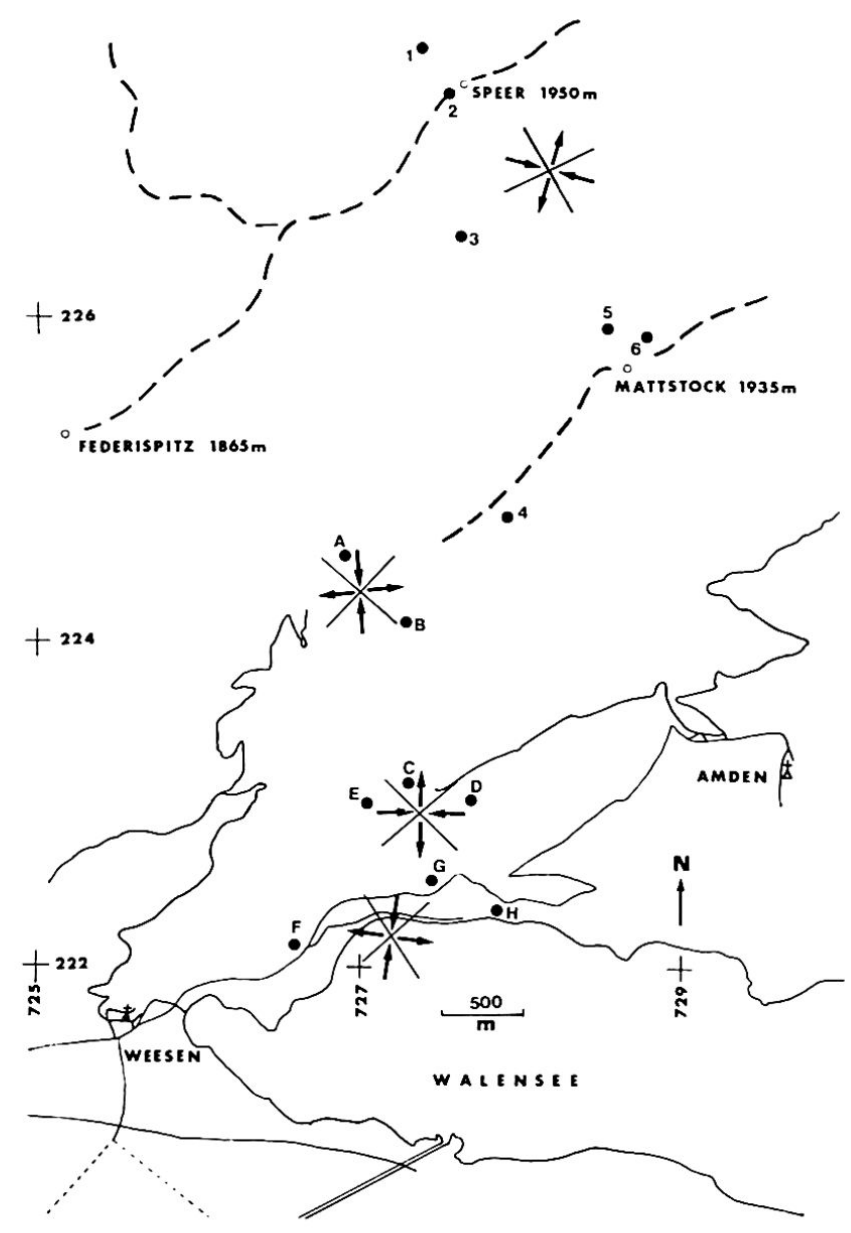

Abb. 6 Resultat der Kluftmessungen im Gebiet um Amden. Die Meßstellen sind mit 1-6 und $\mathrm{A}-\mathrm{H}$ bezeichnet. Die bevorzugten Streichrichtungen der Klüfte in den Gruppen 1-6, AB, $\mathrm{CDE}$ und FGH sind durch gerade Linien bezeichnet, die daraus abgeleiteten Hauptspannungsrichtungen durch Pfeile.

durch die Kluftstellungen ausgedrückten, tektonischen Bedingungen bestehen.

$\mathrm{Da}$ ist einmal die Gruppe der mit Zahlen bezeichneten Aufschlüsse im festen Gebirge des Speers und des Mattstockes (Loc. 1-6). Man erhält hier eine Hauptdruckrichtung in der Richtung N108E, was in etwa, im Rahmen der üblichen lokalen Variationen, der normalen «europäischen» NW-SE gerichteten Hauptdruckrichtung entspricht.

Die mit Buchstaben bezeichneten Aufschlüsse (Loc. A-H) befinden sich im Rutschgebiet. Hier ist eine der Hauptspannungsrichtungen ziemlich genau NS gerichtet. Diese NS-Richtung ist auch die Richtung der Rutschung. Oberhalb der Rutschung (Loc. A \& B) ist die NS gerichtete Hauptspannung ein Druck, am Abriß (Loc. CDE) ein «Zug» (d.h. minimale Druckspannung) und unten an der Straße (Loc. FGH) wieder ein Druck. Man kann diese Umstände so interpretieren, $\mathrm{da} \beta$ man sich vorstellt, daß diese Spannungen lokale natürliche Gebirgsbauspannungen sind: Der Berg «drückt» (wegen seines Gewichtes) hangparallel gegen den Walensee. Am Abbruch findet 
aber wegen der Entlastung eine Spannungsumkehrung statt, so daß die NS-gerichtete Hauptspannung hier zur minimalen Druckspannung wird. Am Fuß der Böschung findet man dann wieder die normalerweise zu erwartende Bauspannung. Die gefundenen Kluftstellungen passen daher sehr gut in das zu erwartende Kräftefeld.

\section{Schlußfolgerungen}

Es kann somit darauf geschlossen werden, daß der Bergrutsch von Amden sich über Jahre vorbereitet hatte. Zuerst erfolgten die Bewegungen als Antwort auf den Regen in der Weise, daß sie jeweilen nach 12 Tagen auf den Regen «ansprachen». In der Endphase aber stiegen die Bewegungsraten exponentiell an, was auf die Ausbildung eines progressiven Bruches hindeutet. Dynamische Studien ergaben, daß die Rutschung genau der allgemeinen Vorhersagekurve folgte und daß ihre Orientationsstruktur dem lokalen neotektonischen Spannungsfelde entsprach.

\section{Dank}

Der Verfasser ist Herrn Dipl. Geol. W. Kyburz vom Geographischen Institut der Universität Zürich zu Dank verpflichtet, da er ihn auf das Problem «Amden» aufmerksam gemacht und geologische Exkursionen alldahin organisiert hat. Herr Prof. Dr. H. Jäckli hat freundlicherweise einige den "Zwischenbericht» ergänzende Daten zur Verfügung gestellt, und das Rechenzentrum der Technischen Universität Wien hat die Rechnungen ausgeführt.

\begin{abstract}
On January 21, 1974, a land slide occurred near Amden in the Canton of St. Gall in Switzerland. This slide is of considerable scientific interest because it had been threatening for some time so that the authorities had arranged for measurements to be made. Thus, creep-curves for the slide during the preparation period are available. These could be compared with the daily rain rates for the nearby meteorological station at Weesen. It was found that in the initial "slow" phase of the slide, the movements
\end{abstract}

were mainly a response to the rainfall; the delay time between the slide velocity and the rain was 12 days. Finally in January, 1974, however, the movement became exponential which indicated the occurrence of a progressive failure. In addition, dynamic studies have been made in the region: The slide conforms exactly to the general prediction curve and its buildup fits entirely into the local geotectonic stress pattern.

\section{Literatur}

Botschaft des Regierungsrates über Maßnahmen zur Behebung der Bergsturzgefahr in Amden vom 20. November 1973. 32 pp. Großer Rat des Kantons St.Gallen, ZI.36.73.01/3973.03.

NEUE ZÜRCHER ZEITUNG, 21. Januar 1974: Warten auf den Bergsturz (Nr. 32, S. 20)

NEUE ZÜRCHER ZEITUNG, 22. Januar 1974: Erster Felssturz bei Amden (Nr. 34, S. 19).

NEUE ZÜRCHER ZEITUNG, 31. Januar 1974: Gewisser Erfolg beim Wassereinsatz im Amdener Bergsturzgebiet (Nr.51, S. 20).

SCHEIDEGGER, A. E., 1973: On the prediction of the reach and velocity of catastrophic landslides. Rock Mechanics, 5, 231-236.

SCHEIDEGGER, A. E., 1977: Kluftmessungen im Gelände und ihre Bedeutung für die Bestimmung des tektonischen Spannungsfeldes in der Schweiz. Geogr. Helv. 32(3), 121-134.

SCHEIDEGGER, A.E., 1984: The significance of surface joints. Geophysical surveys (Reidel) in press.

TAGES-ANZEIGER, 11. Februar 1974: Ob Weesen gab es Nachrutschungen (11.2.74, S. 12).

TAGES-ANZEIGER, 29. Mai 1979: Weesen: 380000 Kubikmeter Erdmasse sind in Bewegung (29. Mai 1979, S. 45).

TAT, 11. Februar 1974: Die "aufgestellte Birne" wurde abgesprengt (Nr. 35, S. 14).

TAT, 22. Februar 1974: Die akute Bergsturzgefahr ist gebannt (Nr. 45, S. 22).

ZÜRICHSEE-ZEITUNG, 12. April 1973: Vorsorgliche Maßnahmen im absturzgefährdeten Gebiet am Walensee (Nr.86, S. 3-4).

ZÜRICHSEE-ZEITUNG, 19. April 1973: Noch keine Gefahr am Walensee (Nr. 92, S. 3).

Zwischenbericht der vorberatenden Kommission über Maßnahmen zur Behebung der Bergsturzgefahr in Amden vom 4. Februar 1974, $7 \mathrm{pp}$. Großer Rat des Kantons St. Gallen, ZI.26.72.01/39.73.03. 